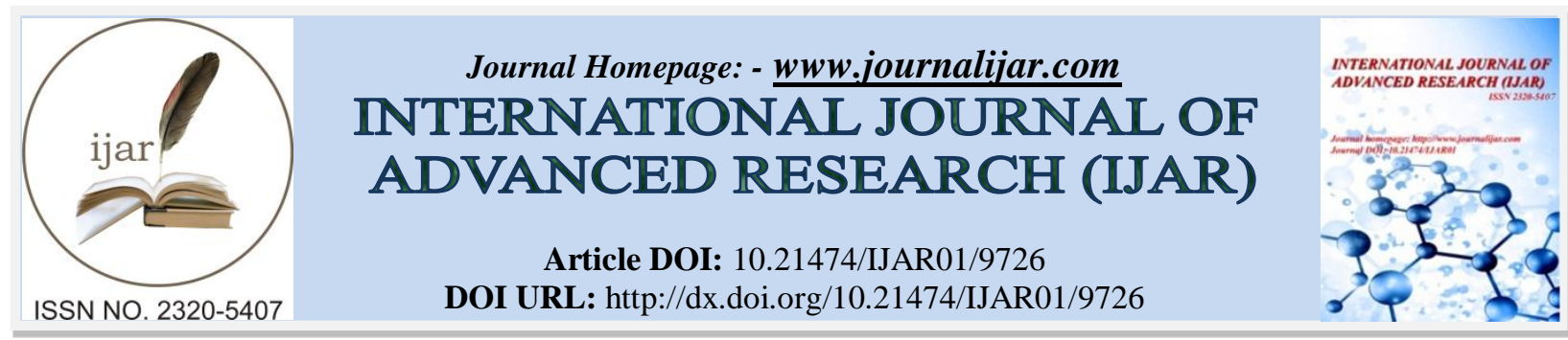

RESEARCH ARTICLE

\title{
ONE ANASTOMOSIS GASTRIC BYPASS AS A SALVAGE MANAGEMENT FOR WEIGHT REGAIN AFTER VERTICAL BANDED GASTROPLASTY; SINGLE INSTITUTE EXPERIENCE.
}

Mohamed Ibrahim ${ }^{1}$, Mohamed Hany ${ }^{2}$, Ahmed Zidan $^{1}$ and Tamer N Abdelbaki ${ }^{2}$.

1. Department of Surgery, Medical Research Institute, Alexandria University, Egypt.

2. Department of Surgery, Faculty of Medicine, Alexandria University, Egypt.

\section{Manuscript Info}

Manuscript History

Received: 09 July 2019

Final Accepted: 12 August 2019

Published: September 2019

Key words:-

Bariatric surgery, revisional bariatric surgery, weight regain, one anastomosis gastric bypass, vertical banded gastroplasty.

\section{Abstract}

Background: Vertical banded gastroplasty (VBG) was a popular bariatric operation two decades ago, but it has been discontinued as it necessitates laparotomy in most cases, and there is often a high incidence of weight regain or insufficient weight loss requiring a revisional bariatric procedure.

Objective: The aim of the present study was to evaluate one anastomosis gastric bypass (OAGB) as a relatively safe and less demanding solution for the management of weight regain after VBG.

Methods: Between February 2014 and September 2017, 42 morbidly obese patients, who had undergone open VBG and suffered from inadequate weight loss or progressive weight regain, were offered laparoscopic OAGB as a revisional surgery. They completed a follow up for at least one year.

Results: All cases were completed laparoscopically. The mean operative time was $166.7 \pm 44.6$ minutes and the mean hospital stay was $2.2 \pm 0.5$ days. The mean BMI before revisional surgery was $47.9 \pm 6.6$ $\mathrm{kg} / \mathrm{m}^{2}$, and after one year of follow up it was $29.2 \pm 4.9 \mathrm{~kg} / \mathrm{m}^{2}$. The mean \%EBMIL was $54.3 \pm 11.1 \%$ at 6 months after surgery and $85.6 \pm 16.2 \%$ at one year after surgery, with improvement of comorbidities observed in most cases.

Conclusion: Laparoscopic OAGB is a safe revisional bariatric option for failed VBG with very successful results in terms of weight reduction, improvement of comorbidities, and low incidence of major complications.

Copy Right, IJAR, 2019,. All rights reserved.

\section{Introduction:-}

Vertical banded gastroplasty (VBG) was a popular bariatric operation two decades ago but it has been discontinued as it necessitates laparotomy in most cases; in addition to its long-term complications, there is a high incidence of weight recidivism and a high incidence of revisional surgery approaching more than 50\%. (1-3)

Weight regain after VBG is related to: staple line disruption, which reduces the element of restriction; proximal pouch enlargement with the formation of a large gastric reservoir of food; ring dilatation between the proximal gastric pouch and the remaining of the stomach with the elimination of restriction; and non-compliance of some patients (changed to sweet eaters). $(2,4)$

Corresponding Author:-Mohamed Ibrahim.

Address:-Department of Surgery, Medical Research Institute, Alexandria University, Egypt. 
The treatment options for failed VBG due to weight regain are variable, including revisional VBG, conversion to Roux En-Y gastric bypass (RYGB), conversion to sleeve gastrectomy (SG), and conversion to one anastomosis gastric bypass (OAGB). (5-7)

Vertical banded gastroplasty (VBG) was previously performed on a relatively large number of morbidly obese patients in Egypt; Buchwald and Oien, (8) in their bariatric surgery worldwide survey done in 2011, reported VBG to be the second most commonly performed bariatric procedure (826 procedures) in Egypt after sleeve gastrectomy (896 procedures). Having had previous success with OAGB at our institute, we performed this study to evaluate the short-term outcome of OAGB as a treatment option for patients with failed VBG.

\section{Patients And Methods:-}

This study was performed at the Department of Surgery in the Medical Research Institute at Alexandria University, Egypt, between February 2014 and September 2017, after approval by the ethical committee of our institute.

We included 42 morbidly obese patients who underwent VBG previously and had inadequate weight loss or progressive weight regain, where the remaining body mass index (BMI) was more than $40 \mathrm{~kg} / \mathrm{m}^{2}$ or more than 35 $\mathrm{kg} / \mathrm{m}^{2}$, respectively. Inadequate weight loss was defined as less than or equal to $50 \%$ of excess weight loss. (9)

All patients were subjected to preoperative evaluation including dietary history assessment, thorough clinical examination, blood chemistry tests, and detailed weight and BMI changes at both pre- and post-VBG as well as after the revisional procedure. All patients were also subjected preoperatively to upper gastrointestinal (GI) endoscopy and virtual computed tomography (CT) gastroscopy with 2D and 3D reformations of the stomach to characterize the gastric anatomy, measure the size of the proximal pouch, and detect staple line disruption (if present) or mesh complications such as erosions (Figure 1 and Table 1).

All patients were prospectively followed for at least 1 year after the revisional OAGB procedure to detect complications, assess changes in weight and BMI, and evaluate improvement of comorbidities.

All patients signed a written informed consent and were counseled about the treatment options prior to surgery.

\section{Surgical Technique (Figures 2-5)}

All operations were performed under general anesthesia with the patient in the reverse Trendelenburg and split leg position. All procedures were attempted laparoscopically; the insertion of the camera port and creation of pneumoperitoneum was done under direct vision by an optical trocar inserted $20 \mathrm{~cm}$ below the xiphoid process and slightly to the patient's left side. The second trocar was inserted in the left axillary line as a working port for the dissection of adhesions between the omentum and the previous abdominal wall scar for safe insertion of other trocars, and to be used later by an assistant for retraction during the procedure. The third and fourth trocars were inserted in the left and right mid-clavicular lines as working ports. The fifth trocar was inserted below the xiphoid process for a liver retraction.

All procedures were started with a full dissection of adhesions between the stomach and liver using the Enseal device (Ethicon Endo-Surgery, Cincinnati, OH, USA), starting at the antrum - which in most cases was a safe area with minimal adhesions - and following this plane up to the angle of His until complete separation of the stomach from the liver was achieved. Special attention was paid to the site of the mesh from the previous VBG; identification of the pouch from the previous VBG was helped by insertion of a 40 Fr calibration bougie into the pouch.

After complete delineation of the VBG pouch, intraoperative upper GI endoscopy was done to measure the distance between the esophago-gastric junction and the upper border of the mesh. This was done to ensure a sufficient length of the future OAGB pouch in order to locate the simple loop gastro-jejunostomy at a minimum or $8 \mathrm{~cm}$ away from the esophago-gastric junction to avoid biliary reflux esophagitis.

Creation of the OAGB pouch within the old VBG pouch started with a transverse stapling using a black reload and an articulating endoscopic linear cutter, Echelon Flex 60 (Ethicon Endo-Surgery, Cincinnati, OH, USA) above the mesh and slightly medial to the old staple line, to allow for drainage of gastric juice of the old VBG pouch. This was followed by vertical stapling up to the angle of His, using black or green reloads guided by the 40 Fr bougie to 
complete the new OAGB pouch. The staple line was reinforced by invaginating sutures using 3/0 V-Loc 180 sutures (Covidien, Mansfield, MA, USA).

The remnant stomach was left if it appeared healthy after dissection of the adhesions and if there was good drainage of the old VBG pouch without stapling across the old staples. We controlled bleeding from the staple line with clips. After the pouch was formed, a stapled simple loop gastrojejunostomy was done at two meters distal to the ligament of Treitz using a blue reload in an antecolic fashion, and closure of the enterotomy and gastrotomy done in double layers using 3/0 V-Loc 180 sutures (Covidien, Mansfield, MA, USA).

Intraoperative upper GI endoscopy was used again at the end of the procedure to visualize the mucosa of the pouch to ensure no presence of ischemia, to evaluate the integrity of the pouch and the anastomosis (Figure 6), and to perform a test for leakage by air insufflation under water seal. A test for leakage using methylene blue was also done in all cases to test the anastomosis. No drains nor nasogastric tubes were left at the end of the operations in all cases.

\section{Postoperative Course}

All patients were observed by well-trained nursing staff; vital signs were continuously monitored. All patients had venous thrombo-embolism (VTE) prophylaxis by enoxaparin and were encouraged to start mobilization as early as possible.

Oral fluids were started at a rate of 2-3 liters per day for 1 week, followed by introduction of soft diet as consulted by a dietitian. Patients were discharged from the hospital in cases of adequate oral fluid intake without vomiting, together with normal vital signs and the absence of any other discomfort.

All patients with suspicious symptoms and signs (e.g. tachycardia, fever, persistent vomiting, and persistent abdominal or left shoulder pain) were administered a CT study with oral gastrografin and IV contrast to ensure there were no leaks.

\section{Outcome Measurement And Follow-Up}

The clinical data were prospectively collected and included age, gender, body mass index (BMI) at the primary surgery and at revisional OAGB, the indications for revision, operative time, hospital stay, early morbidity, and mortality.

Operative data were recorded, including: operative time, intraoperative complications, and associated procedures, if any.

Patients were followed up at one, six, and 12 postoperative months and then annually thereafter.

Postoperative work-up included: recording of early and late postoperative complications; and the effect of the operation on weight loss, calculated at six months and at one year after surgery in the form of percentage excess BMI loss (\%EBMIL) using the following formula: ([operative BMI - follow-up BMI] $\times 100$ ) divided by (operative BMI - 25).

\section{Statistical Analysis:-}

Data was analyzed using IBM SPSS software package version 20.0. (Armonk, NY: IBM Corp). ANOVA with repeated measures and post-hoc test (Bonferroni) was applied to normally distributed quantitative variables for comparison between different periods. A p-value of $<0.05$ was deemed statistically significant.

\section{Results:-}

This study included 42 patients who fulfilled at least 1 year of follow up after revisional surgery. The mean age was $43.9 \pm 7.4$ years. The indication for surgery was due to weight regain in 35 cases $(83.3 \%)$ and inadequate weight loss in 7 cases $(16.7 \%)$ (Table 2).

The mean BMI before initial surgery and before revisional surgery was $47.5 \pm 3.9 \mathrm{Kg} / \mathrm{m} 2$ and $47.9 \pm 6.6 \mathrm{Kg} / \mathrm{m} 2$, respectively, and the mean duration between the initial VBG and revisional OAGB surgery was $9.0 \pm 1.4$ years (Table 2). 
In three cases $(7.14 \%)$ with complete staple line disruption of the previous VBG pouch, we formed the pouch below the mesh as guided by intraoperative endoscopy. In one case $(2.38 \%)$ with staple line disruption, there was mesh erosion and vertical stapling was helped by the endoscope to avoid accidental stapling of the eroded intra-gastric mesh (Figure 7).

In seven cases $(16.66 \%)$, we resected the remaining stomach until the level below the mesh to avoid stapling across the old staple line of the previous VBG during the formation of the OAGB pouch and to prevent formation of a blind pouch with a resulting mucocele in the remnant stomach.

In two cases, the pouch of the stomach was very small due to high insertion of the mesh in one case and pouch perforation due to dissection in the other case. The resulting small pouches in these cases were not suitable for OAGB, so we instead performed RYGB and these cases were excluded from the study.

Incisional hernia was present in four cases $(9.52 \%)$ and was repaired after the definitive procedure was completed, and combined cholecystectomy and OAGB was performed in six cases (14.28\%).

The mean operative time was $166.7 \pm 44.6$ minutes and the mean hospital stay was $2.2 \pm 0.5$ days (Table 2).

Minor complications in the form of wound infection were present in five cases $(11.9 \%)$ and were treated with local antibiotics. Mild reflux symptoms were present in seven cases (16.66\%) during follow up and were treated with prokinetics and proton pump inhibitors.

Major leakage complications were present in one case $(2.38 \%)$ and the patient was readmitted at postoperative day five with persistent fever, abdominal pain and left shoulder pain; CT abdomen with oral gastrografin was ordered and revealed foci of pneumo-peritoneum and a minimal to mild collection at the surgical bed. Laparoscopic exploration was performed and the site of leakage was identified at the angle between the afferent limb and the OAGB pouch. Abdominal lavage was performed and repair sutures with Vicryl $3 \backslash 0$ were used to reinforce the anastomosis at that site with the insertion of an intra-abdominal drain at the anastomotic site. However, the patient did not improve and the drain output was of an intestinal nature; the patient was returned to the operative room for another laparoscopic exploration which revealed leakage from the same point, mostly due to high tension. We disconnected the afferent limb of the intestine from the stomach and performed entero-enteric anastomosis with the efferent limb to make a Roux en Y gastrojejunostomy. We then inserted $18 \mathrm{~cm}$ long fully covered self-expandable metallic stents, SEMS (Niti-S Mega esophageal stent, Taewoong Medical, Gyeonggi-do, South Korea) endoscopically to cover the anastomosis traversing between the esophagus, stomach pouch, and the efferent limb. The patient improved and was discharged from the hospital after stabilization and radiological confirmation of the stent in place without active leakage. The stent was removed after six weeks; an abdomen CT was performed and revealed no active leak after stent removal, and the patient recovered to normalcy thereafter.

Regarding weight loss and BMI changes along the period of follow up, there was a statistically significant BMI loss at six months and after one year of surgery in comparison to the BMI before the revisional OAGB (Table 3).

There was also a significant improvement of comorbidities after the revisional surgery: two patients (4.76\%) who were diabetic were cured one year after surgery; three patients $(7.14 \%)$ with hypertension were cured $(2 / 3)$ or improved with good control on anti-hypertensive drugs (1/3); 12 patients $(28.57 \%)$ with osteoarthritis were cured; two patients $(4.76 \%)$ with dyslipidemia were cured; and one patient $(2.38 \%)$ with fertility issues was able to get pregnant 14 months after surgery.

\section{Discussion:-}

VBG was one of the most popular bariatric procedures in the early 1990s because it was a relatively simple and safe restrictive bariatric procedure which avoided the complications of malabsorptive procedures. (10) Although the early outcome regarding weight loss was encouraging, the long-term results were unsatisfactory, with the failure rate reaching up to $79 \%$ of cases. (11)

Revisional bariatric surgery is well known to be a difficult task with a high incidence of major complications due to altered anatomy and dense adhesions. (12) Many options are available for revisional bariatric procedures due to 
weight regain after VBG, such as conversion to RYGB, but it is a challenging option with relatively high morbidity and mortality. (13)

The earliest results of OAGB were published by Rutledge in 2001, and OAGB has since been used as a primary and revisional procedure for morbid obesity. (14-16) In a comparative study between RYGB and OAGB by Lee et al. (17), the authors found that weight loss was greater in the OAGB group after a 10 year follow up.; in addition, the complication rate was lower in the OAGB group with no significant difference in comorbidities improvement.

In this study, we have found a safe strategy for the management of failed VBG due to weight recidivism to address a growing number of patients seeking surgery for their morbid obesity status. We evaluated OAGB as revisional VBG procedure and found it to be a relatively safe and less demanding solution for management of weight recidivism after VBG.

The present study included 42 patients. Female patients represented the majority of the cases (85.7\%) in our study as well as in similar published studies, perhaps because female patients are more concerned about their physical appearance than men $(18,19)$.

In this study, we formed the pouch above the mesh, except in cases with complete staple line disruption, and medial to the previous VBG stapling to avoid the previous staple line as much as possible, with invagination achieved by continuous seromuscular sutures to reinforce the staple line. Wang et al. (18) performed their revisional OAGB after laparoscopic VBG in 21 cases, and in 8 cases with open VBG, they started the pouch at the level of the antrum below the crow's foot, removing the silastic ring from VBG in cases of a stenotic ring with stapling across the old staple line.

Noun et al. (16) published their study of 17 cases with failed open VBG, including two cases of revisional VBG. Their approach was to open through the previous scar and dissection was limited to the distal portion of the stomach; if present, the silastic ring was removed in all cases with staple line reinforcement.

In another study published on 2016 by Salama and Sabry (19) comparing OAGB and RYGB for revision after failed VBG, the authors formed the pouch below the mesh if there was no outlet obstruction with vertical stapling at the previous VBG stable line. In the case of outlet obstruction, they tried to remove the mesh; however, if it was difficult to remove, stapling was started above the mesh and proceeded as RYGB (if the pouch size was adequate). We have two cases in the present study for which we were obliged to perform RYGB due to a short pouch, as previously mentioned.

The mean operative time in the present study was $166.7 \pm 44.6$ minutes and the mean hospital stay was $2.2 \pm 0.5$ days. The studies by Wang et al. (18) and Noun et al. (16) reported mean operative times of $171.4 \pm 15.3$ minutes and 184.76 minutes respectively, and mean hospital stays of $6.4 \pm 3.2$ days and 5.27 days, respectively.

In the present study, we had one case of leakage at the site between the afferent limb and the OAGB pouch, which led us to perform an interrupted hypnotic stitch in order to support the anastomosis at that site. Wang et al. (18) reported one case with upper gastrointestinal bleeding from the staple line which was treated conservatively, one case with small bowel obstruction due to port site hernia, and one mortality due to leakage and sepsis, raising the importance of close follow up of the patients.

Noun et al. (16) reported two cases with intra-abdominal abscess treated by percutaneous drainage. Salama and Sabry (19) had one case of leakage out of 39 cases due to iatrogenic injury to the ascending limb of the small intestine, which was repaired immediately.

Regarding weight loss, this study showed that OAGB was very effective as the mean BMI before revisional surgery was $47.9 \pm 6.6 \mathrm{~kg} / \mathrm{m}^{2}$ and after one year of follow up it was $29.2 \pm 4.9 \mathrm{Kg} / \mathrm{m} 2$. Furthermore, the mean \%EBMIL was $54.3 \pm 11.1 \%$ at six months after surgery and $85.6 \pm 16.2 \%$ at one year after surgery.

Wang et al. (18) reported a mean BMI of $32.1 \mathrm{Kg} / \mathrm{m} 2$ after one year as compared with that before revisional surgery $(\mathrm{P}<0.001)$. Salama and Sabry (19) published that mean BMI was dropped significantly at one year after OAGB with no significant difference between OAGB and RYGB $(\mathrm{P}=0.654)$. 


\section{Conclusion:-}

Revisional bariatric surgery after open VBG is a demanding procedure; conversion to OAGB is a safe procedure with very successful results in both weight reduction and comorbidities improvement and has a low incidence of major complications.

\section{Conflict of interest}

Drs. Ibrahim, Hany, Zidan and abdelbaki have no conflicts of interest or financial ties to disclose.

\section{Informed consents}

All patients signed an informed consent and were counseled about the treatment options.

\section{Ethical approval}

The study was approved from our institutional ethical committee.

Table 1:-Mean data of VBG pouch by virtual CT gastroscopy

\begin{tabular}{|l|l|}
\hline Volume of pouch $(\mathrm{cc})$ & $75.7 \pm 18.2$ \\
\hline Size of stoma $(\mathrm{cm})$ & $1.2(0.5-2.0)$ \\
\hline Mesh erosion $(\mathrm{n})$ & $3(7.1 \%)$ \\
\hline Staple line disruption(n) & $3(7.1 \%)$ \\
\hline
\end{tabular}

Table 2:-Demographic data of cases included in the study $(\mathrm{n}=42)$

\begin{tabular}{|l|l|}
\hline Sex & No. $(\%)$ \\
\hline Male & \\
\hline Female & $6(14.3 \%)$ \\
\hline Mean Age (years) & $36(85.7 \%)$ \\
\hline Reason for revision & $43.9 \pm 7.4$ \\
\hline$\quad$ Weight regain & \\
\hline Insufficient weight loss & $35(83.3 \%)$ \\
\hline Mean duration since previous VBG (years) & $7(16.7 \%)$ \\
\hline Mean operative Time (minutes) & $9.0 \pm 1.4$ \\
\hline Mean hospital stay (days) & $166.7 \pm 44.6$ \\
\hline Mean duration of follow up (months) & $2.2 \pm 0.5$ \\
\hline Mean BMI changes: & $26.5(12.0-48.0)$ \\
\hline Initial mean BMI $(\mathrm{kg} / \mathrm{m} 2)$ & \\
\hline Least mean BMI $(\mathrm{kg} / \mathrm{m} 2)$ & $47.5 \pm 3.9$ \\
\hline Present mean BMI $(\mathrm{kg} / \mathrm{m} 2)$ & $35.5 \pm 3.9$ \\
\hline
\end{tabular}

Table 3:-Distribution of BMI and \%EBMIL over the follow up period $(\mathrm{n}=42)$

\begin{tabular}{|c|c|c|c|c|c|c|}
\hline 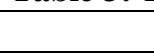 & \multirow[t]{2}{*}{ Initial } & \multirow[t]{2}{*}{ Least } & \multirow[t]{2}{*}{ Present } & \multicolumn{2}{|l|}{ Follow up } & \multirow{2}{*}{$\mathrm{p}$} \\
\hline & & & & $6 \mathrm{~m}$ & $1 \mathrm{y}$ & \\
\hline BMI & $47.5 \pm 3.9$ & $35.5 \pm 3.9$ & $47.9 \pm 6.6$ & $36.0 \pm 5.4$ & $29.2 \pm 4.9$ & $<0.001^{*}$ \\
\hline$\%$ EBMIL & - & - & - & $54.3 \pm 11.1$ & $85.6 \pm 16.2$ & $<0.001^{*}$ \\
\hline
\end{tabular}

N.B.Statistically significant at $\mathrm{p} \leq 0.05$ 


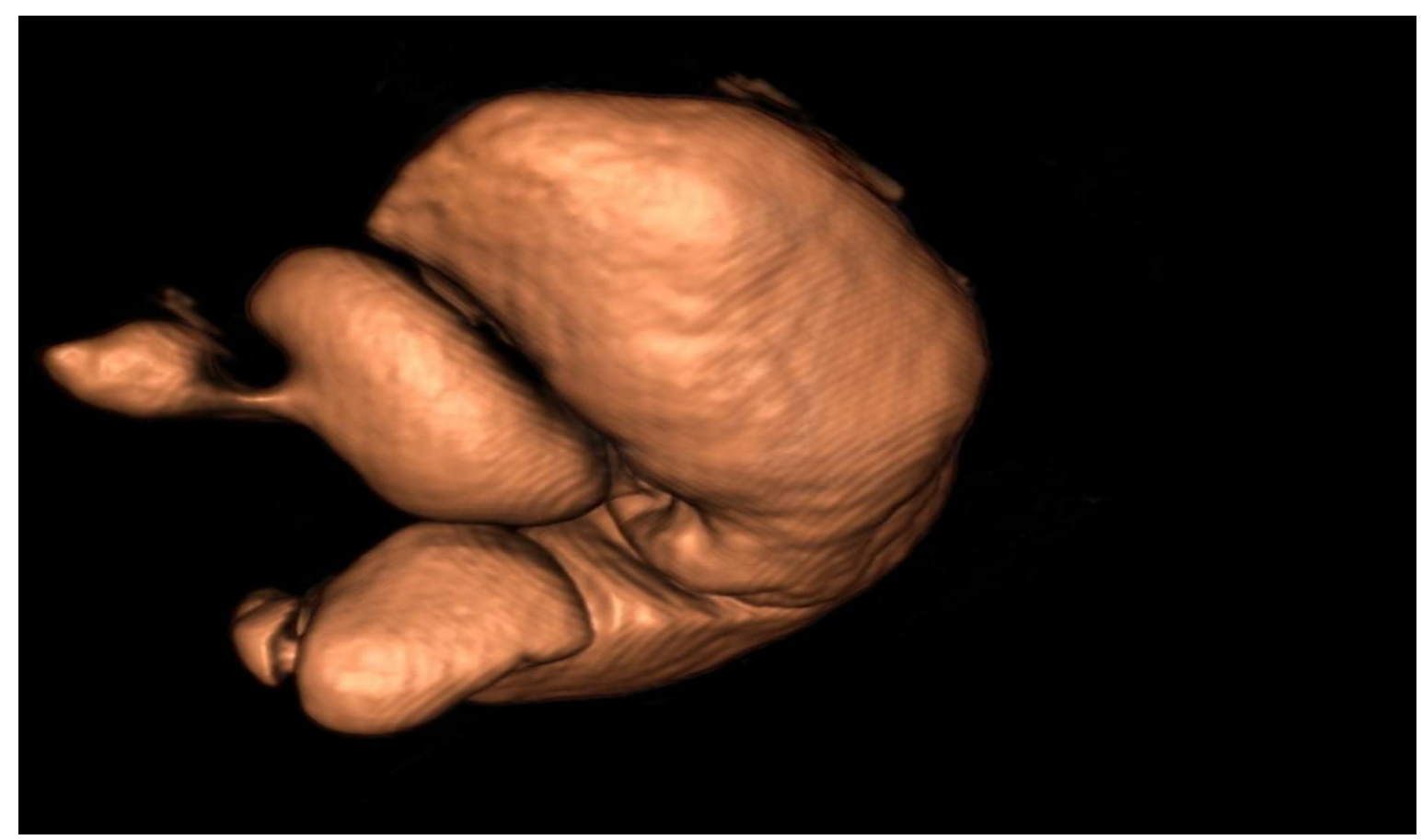

Figure 1:-Virtual CT Gastroscopy for a stomach with a previous VBG

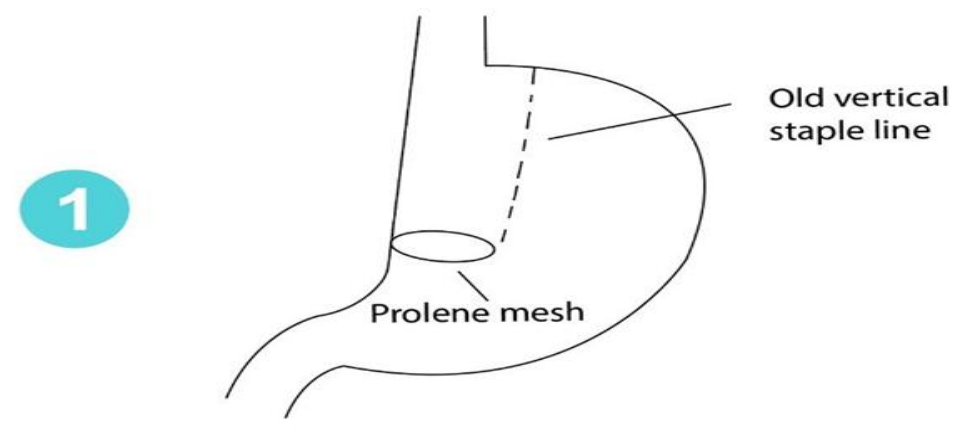

Figure 2:-The pouch of the previous VBG. 


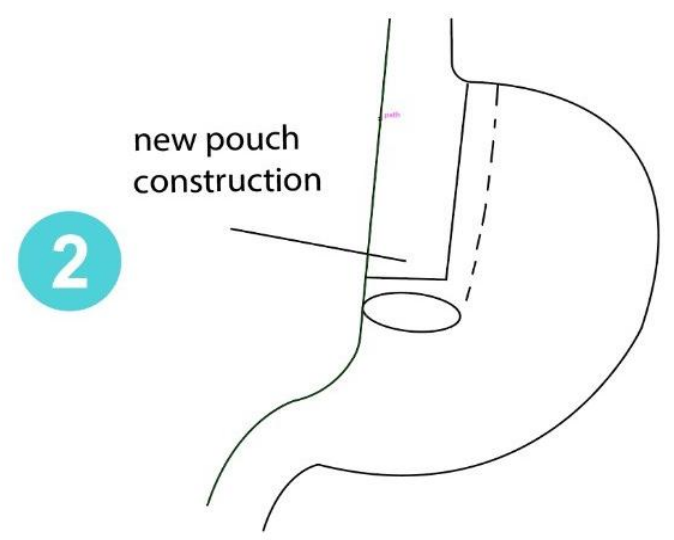

Figure 3:-the design of the OAGB pouch within the previous VBG pouch.

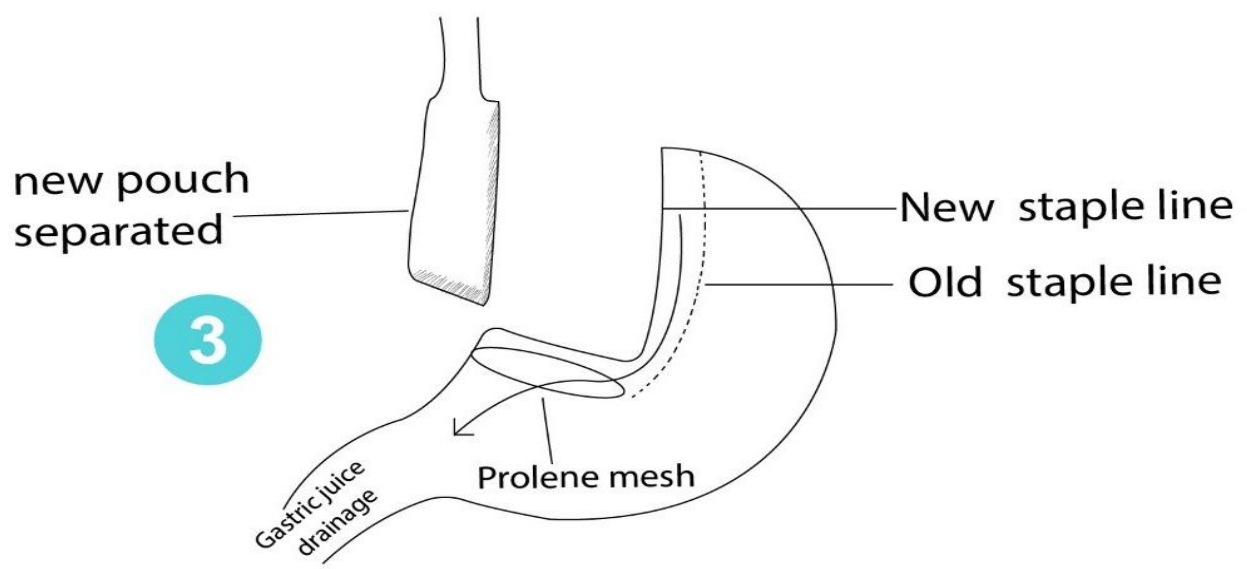

Figure 4:-Creation of OAGB pouch completed.

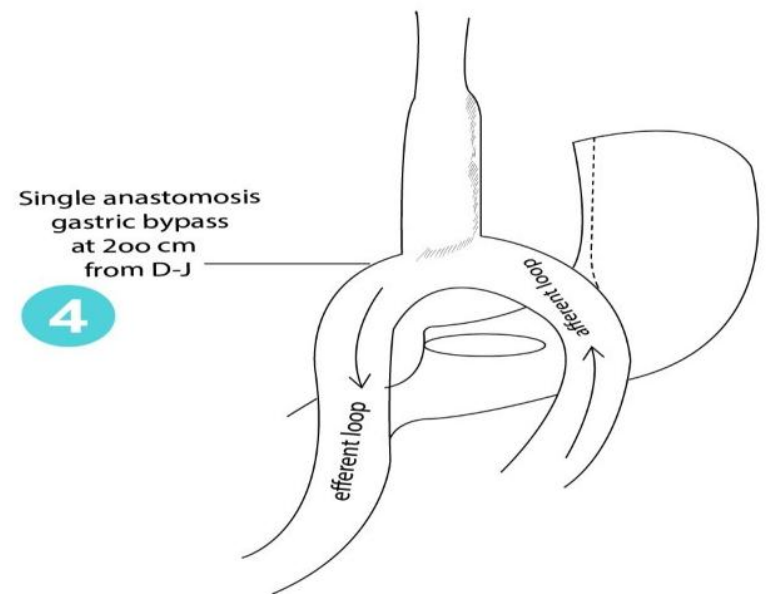

Figure 5:-Completed OAGB procedure. 


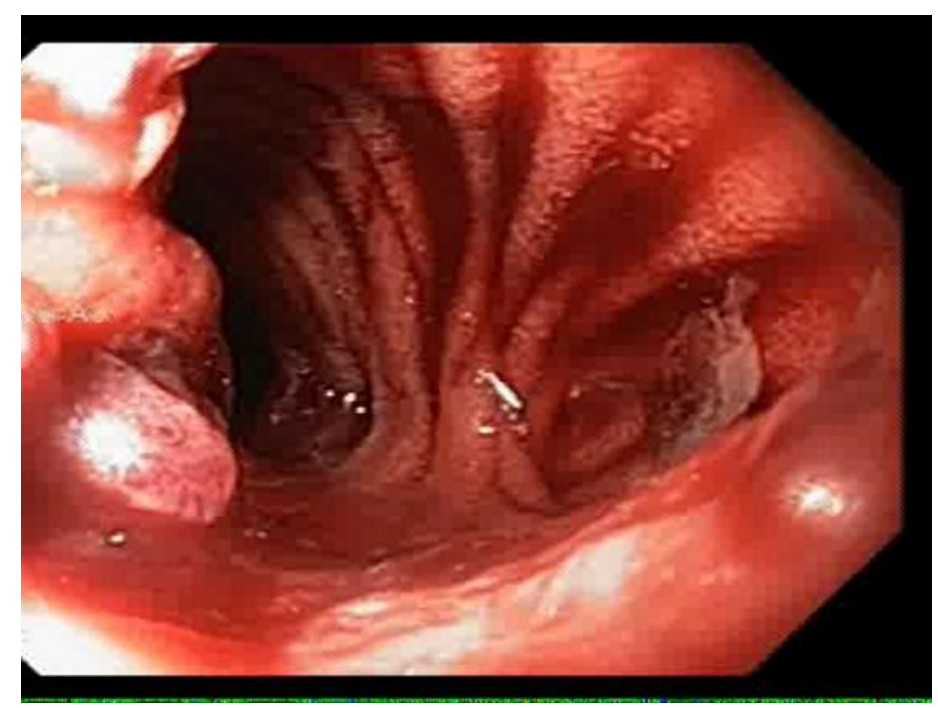

Figure 6:-intra-operative endoscopic view of OAGB.

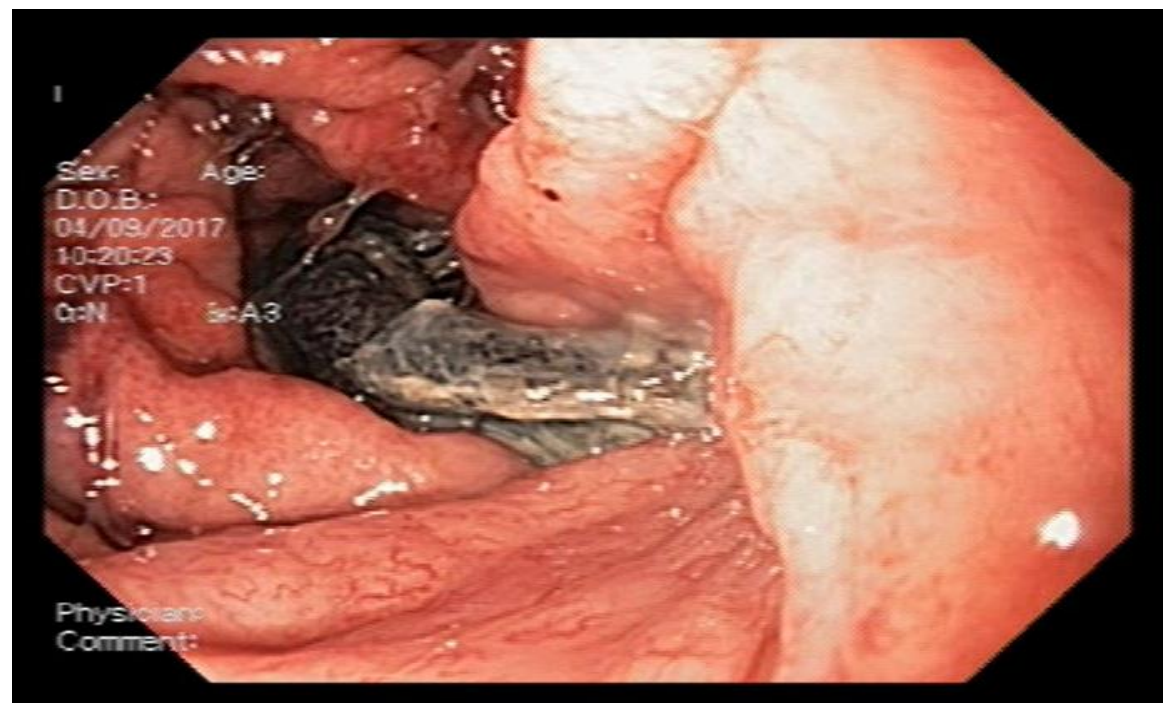

Figure 7:-Upper GI endoscopy showing eroded mesh of a previous VBG

\section{References:-}

1. Van Gemert W, Van Wersch M, Greve J, Soeters P. Revisional surgery after failed vertical banded gastroplasty: restoration of vertical banded gastroplasty or conversion to gastric bypass. Obesity Surgery. 1998;8(1):21-8.

2. Kaminski D. Gastric restrictive procedures to treat obesity: reasons for failure and long-term evaluation of the results of operative revision. International journal of surgical investigation. 2001;2(5):413-21.

3. Schouten R, Wiryasaputra DC, Van Dielen FM, Van Gemert WG, Greve JWM. Long-term results of bariatric restrictive procedures: a prospective study. Obesity surgery. 2010;20(12):1617-26.

4. Gagner M, Gumbs AA. Gastric banding: conversion to sleeve, bypass, or DS. Surgical endoscopy. 2007;21(11):1931-5.

5. Iannelli A, Schneck AS, Ragot E, Liagre A, Anduze Y, Msika S, et al. Laparoscopic sleeve gastrectomy as revisional procedure for failed gastric banding and vertical banded gastroplasty. Obesity surgery. 2009;19(9):1216-20.

6. Suter M, Ralea S, Millo P, Allé J-L. Laparoscopic Roux-en-Y gastric bypass after failed vertical banded gastroplasty: a multicenter experience with 203 patients. Obesity surgery. 2012;22(10):1554-61.

7. Vasas P, Dillemans B, Van Cauwenberge S, De Visschere M, Vercauteren C. Short-and long-term outcomes of vertical banded gastroplasty converted to Roux-en-Y gastric bypass. Obesity surgery. 2013;23(2):241-8.

8. Buchwald H, Oien DM. Metabolic/bariatric surgery worldwide 2011. Obesity surgery. 2013;23(4):427-36. 
9. Magro DO, Geloneze B, Delfini R, Pareja BC, Callejas F, Pareja JC. Long-term weight regain after gastric bypass: a 5-year prospective study. Obesity surgery. 2008;18(6):648-51.

10. Mason EE, Director I. Change in obesity surgery. Obes Surg. 1997;7:373-4.

11. Balsiger BM, Poggio JL, Mai J, Kelly KA, Sarr MG. Ten and more years after vertical banded gastroplasty as primary operation for morbid obesity. Journal of Gastrointestinal Surgery. 2000;4(6):598-605.

12. Roller JE, Provost DA. Revision of failed gastric restrictive operations to Roux-en-Y gastric bypass: impact of multiple prior bariatric operations on outcome. Obesity surgery. 2006;16(7):865-9.

13. Fernandez Jr AZ, Demaria EJ, Tichansky DS, Kellum JM, Wolfe LG, Meador J, et al. Multivariate analysis of risk factors for death following gastric bypass for treatment of morbid obesity. Annals of surgery. 2004;239(5):698.

14. Rutledge R. The mini-gastric bypass: experience with the first 1,274 cases. Obesity surgery. 2001;11(3):276-80.

15. Rutledge R, Walsh TR. Continued excellent results with the mini-gastric bypass: six-year study in 2,410 patients. Obesity surgery. 2005;15(9):1304-8.

16. Noun R, Zeidan S, Riachi E, Abboud B, Chalhoub V, Yazigi A. Mini-gastric bypass for revision of failed primary restrictive procedures: a valuable option. Obesity surgery. 2007;17(5):684.

17. Lee W-J, Ser K-H, Lee Y-C, Tsou J-J, Chen S-C, Chen J-C. Laparoscopic Roux-en-Y vs. mini-gastric bypass for the treatment of morbid obesity: a 10-year experience. Obesity surgery. 2012;22(12):1827-34.

18. Wang W, Huang M-T, Wei P-L, Chiu C-C, Lee W-J. Laparoscopic mini-gastric bypass for failed vertical banded gastroplasty. Obesity surgery. 2004;14(6):777-82.

19. Salama T, Sabry K. Redo Surgery after Failed Open VBG: Laparoscopic Minigastric Bypass versus Laparoscopic Roux en Y Gastric Bypass_-Which Is Better? Minimally invasive surgery. 2016;2016. 